\title{
HEPATOJEJUNOSTOMIA PERIFÉRICA: UMA ALTERNATIVA TÉCNICA NO TRATAMENTO PALIATIVO DAS NEOPLASIAS MALIGNAS DOHILO HEPÁTICO
}

\author{
PERIPHERAL HEPATOJEJUNOSTOMY: A TECHNIC OPTION FOR PALIATIVE \\ TREATMENT OF HILAR CANCER
}

\author{
Roberto Pelegrini Coral'; Natalino Rinaldi²; \\ Guilherme Fagundes Bassols²; Pedro Reck dos Santos ${ }^{3}$; Kelly Ribeiro Neves ${ }^{4}$
}

\begin{abstract}
RESUMO: Objetivo: Relatar a experiência do nosso Serviço com a descompressão cirúrgica da árvore biliar através de uma hepatojejunostomia periférica. Método: Entre julho de 2000 a julho de 2005, 11 pacientes foram à laparotomia para ressecção de tumores do hilo hepático e, durante o trans-operatório, apresentavam lesões irressecáveis. Os dados analisados foram: idade, sexo, morbidade, mortalidade, dosagem de bilirrubinas séricas pré-operatórias e no $7^{\circ}$ dia de pós-operatório, prurido pré e pós-operatório e sobrevida. Resultados: A idade média dos pacientes foi de 67 anos, seis eram mulheres e cinco eram homens. Icterícia estava presente em $100 \%$ dos casos e prurido em $80 \%$. Seis pacientes tiveram o diagnóstico de neoplasia de vesícula biliar e cinco de colangiocarcinoma. Ocorreram três óbitos intra-hospitalares. A dosagem média no pré-operatório de bilirrubina total foi 19,33mg/ dl e bilirrubina direta $16,81 \mathrm{mg} / \mathrm{dl}$ e no pós-operatório $4,88 \mathrm{mg} / \mathrm{dl}$ e $3,64 \mathrm{mg} / \mathrm{dl}$, respectivamente. Oito pacientes que receberam alta hospitalar tiveram sobrevida média de oito meses, evoluindo sem icterícia e prurido. Conclusão: A hepatojejunostomia periférica pode ser considerada uma boa opção como tratamento paliativo. Ela demonstrou ser segura, com sangramento mínimo, rápida execução e mortalidade aceitável, melhorando significativamente a icterícia, o prurido e fornecendo uma sobrevida satisfatória (Rev. Col. Bras. Cir. 2008; 35(3): 173-176).
\end{abstract}

Descritores: Fígado/cirurgia; Neoplasias hepáticas; Assistência paliativa.

\section{INTRODUÇÃO}

O tratamento das neoplasias malignas hilares hepáticas com melhores resultados é a ressecção tumoral ampla ${ }^{1-3}$ Em doença irressecável, estão indicadas terapêuticas paliativas para a icterícia ${ }^{1-4}$. A drenagem endoscópica com Stent e a percutânea trans-hepática estão sujeitas à obstrução das próteses, colangite, insatisfação do paciente, sangramento e necessidade de novas drenagens ${ }^{1,3}$. As abordagens cirúrgicas paliativas têm resultados variáveis e consistem em derivações biliares convencionais com anastomoses dos ductos biliares extra-hepáticos ou intra-hepáticos com o jejuno ${ }^{2,4,5}$.

Relatamos a experiência do nosso Serviço com a anastomose do parênquima hepático com o jejuno que passaremos a denominá-la de hepatojejunostomia periférica. Analisamos as características dos pacientes, os aspectos intra e pós-operatórios, alívio da icterícia, prurido e sobrevida.

\section{MÉTODO}

Observou-se, entre julho de 2000 e julho de 2005, 11 pacientes submetidos à laparotomia para ressecção de tumores hilares hepáticos que, durante o trans-operatório, apre- sentavam lesões irressecáveis. Todos os pacientes foram abordados pela incisão subcostal bilateral (Chevron) e após definição de irressecabilidade, realizava-se hepatectomia segmentar ("Crushing Technique") com preservação dos ductos biliares dilatados.(figura1) Realizaram-se anastomoses do

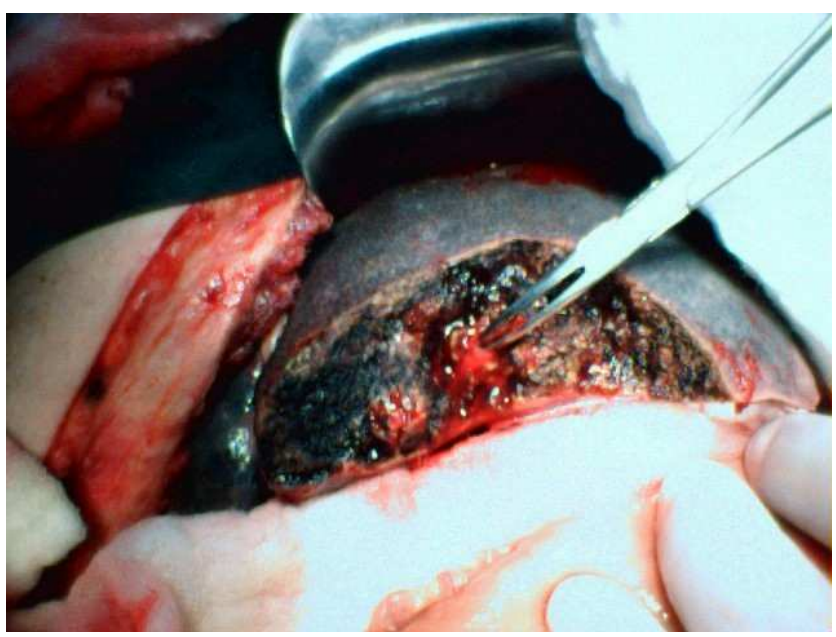

Figura 1 - Preparação do leito hepático e visualização dos canalículos dilatados.

\footnotetext{
1. Professor de cirurgia digestiva da Universidade Luterana do Brasil e Chefe do Serviço de Cirurgia Geral da Irmandade Santa Casa de Misericórdia de Porto Alegre.

2. Cirurgião Geral da Irmandade Santa Casa de Misericórdia de Porto Alegre.

3. Residente de Cirurgia Geral da Irmandade Santa Casa de Misericórdia de Porto Alegre.

4. Doutoranda da Irmandade Santa Casa de Misericórdia de Porto Alegre.

Recebido em 03/12/2007

Aceito para publicação em 31/01/2008

Conflito de interesses: nenhum

Fonte de financiamento: nenhuma

Trabalho realizado na Irmandade Santa Casa de Misericórdia de Porto Alegre.
} 
jejuno com o segmento III do fígado em $45 \%$,(Figuras 2 e 3 ) com o VI em $18 \%$ e combinadas nos segmentos III e VI em $36 \%$ dos pacientes(Figura 4). Utilizou-se fio monofilamentar inabsorvível de polipropileno 3-0 em sutura contínua entre o parênquima hepático e a alça intestinal em Y-Roux e posicionado dreno de Penrose por sete dias. Em dois pacientes que apresentavam invasão duodenal realizou-se uma gastroenteroanastomose , além da hepatojejunostomia periférica.

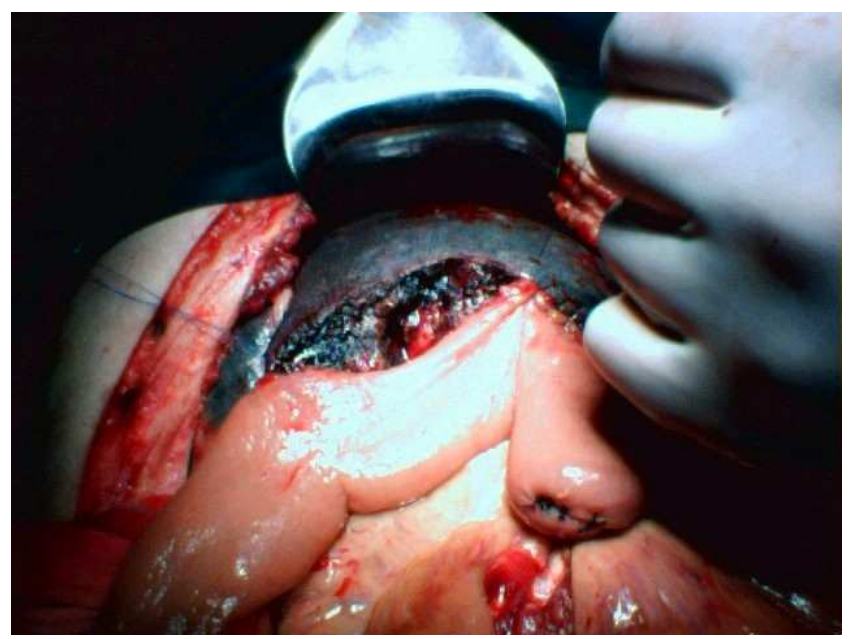

Figura 2 - Plano posterior da anastomose com o jejuno.

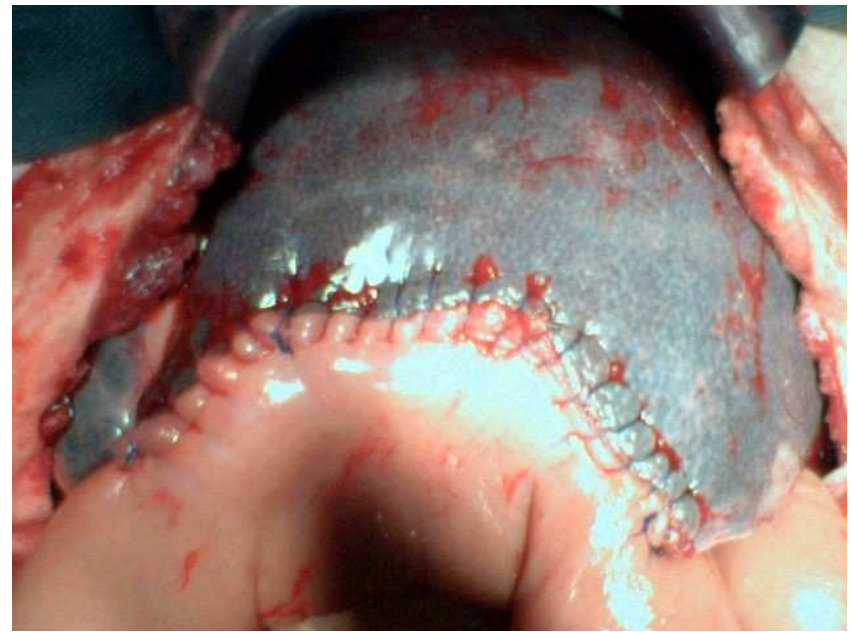

Figura 3 - Hepatojejunostomia periférica unilateral. Aspecto final.

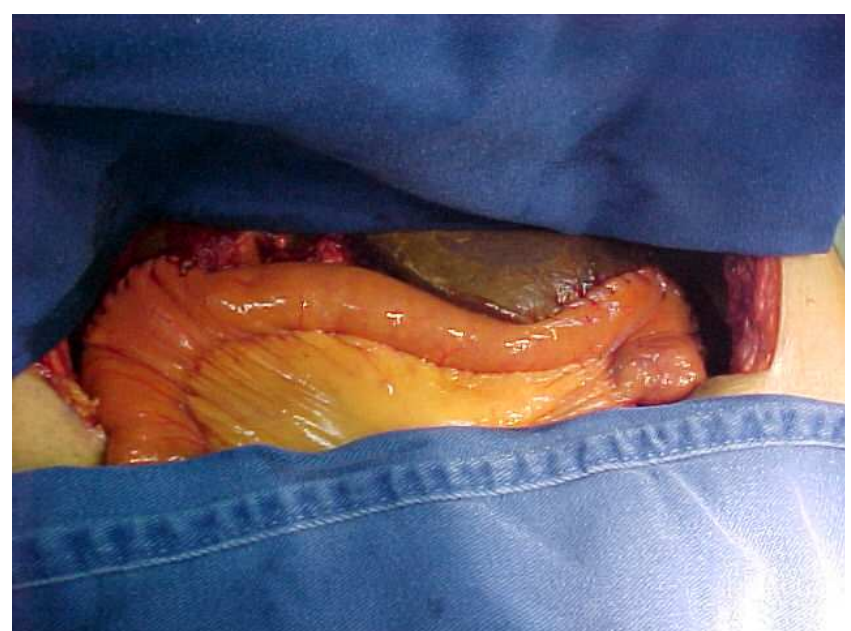

Figura 4 - Hepatojejunostomia periférica bilateral. Aspecto final.
Analisaram-se idade, sexo, morbidade, mortalidade, bilirrubinas séricas pré-operatórias e no $7^{\circ}$ dia de pós-operatório, prurido pré e pós-operatório. Utlizou-se o contato telefônico para a verificação de sobrevida.

\section{RESULTADOS}

Os pacientes tinham idade média de 67 anos, seis eram mulheres e cinco eram homens. A icterícia estava presente em $100 \%$ dos casos, prurido e emagrecimento em $80 \%$ e dor abdominal em $45 \%$. Seis pacientes tiveram o diagnóstico de neoplasia de vesícula biliar e cinco de colangiocarcinoma. O sangramento médio intra-operatório foi $350 \mathrm{ml}$ e cinco pacientes necessitaram de transfusão, com média de uma unidade de concentrado de hemácias por paciente. $\mathrm{O}$ tempo médio cirúrgico foi de quatro horas. Não constatamos fístulas pós-operatórias. Ocorreram três óbitos intra-hospitalares, dois por broncopneumonia, no $5^{\circ}$ e no $9^{\circ}$ dias de pós-operatório, e um por acidente vascular encefálico de tronco cerebral no $8^{\circ} \mathrm{PO}$. No pré-operatório, a média de bilirrubina total foi $19,33 \mathrm{mg} / \mathrm{dl}$ e bilirrubina direta 16,81 $\mathrm{mg} / \mathrm{dl}$ e no pós-operatório foi 4,88 mg/dl e 3,64 mg/dl, respectivamente (gráfico 1). Oito pacientes com alta hospitalar evoluíram sem prurido (gráfico 2). Um paciente submetido à drenagem do segmento III desenvolveu colangite em dois meses, foi reoperado e submetido à drenagem do ducto IV para a mesma alça. Teve boa recuperação, evoluindo sem mais episódios de colangite. Dentre aqueles com alta hospitalar, a sobrevida média foi de oito meses (gráfico 3).

\section{DISCUSSÃO}

A hepatojejunostomia periférica pode melhorar a qualidade de vida e ser comparável às técnicas não-cirúrgicas. Possibilita a drenagem biliar em ponto distante do tumor com menor chance de invasão neoplásica da anastomose ${ }^{1,3}$.

As drenagens não-cirúrgicas possuem risco de obstrução, migração das próteses e necessidade de novos procedimentos para desobstrução ou recolocação ${ }^{3}$. Geralmente, não

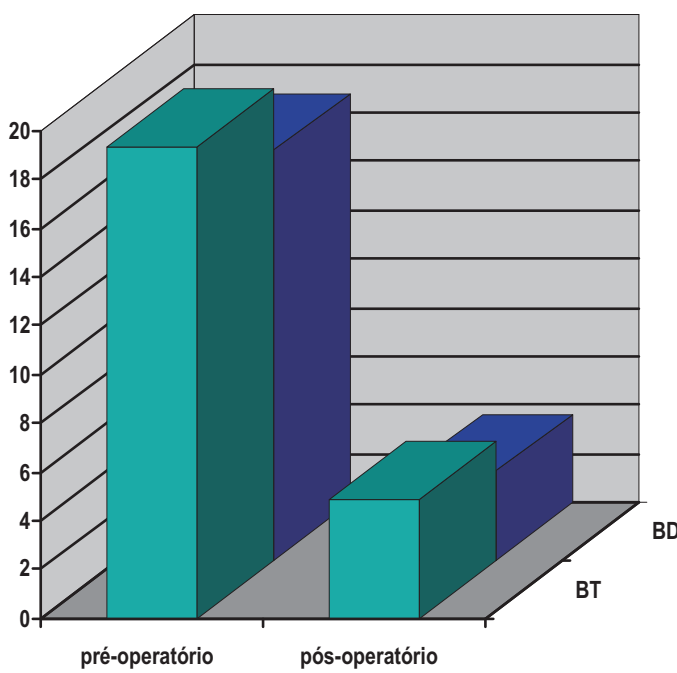

$\square \mathrm{BT}$

Gráfico1 - Níveis de Bilirrubinas pré e pós-operatórias. 


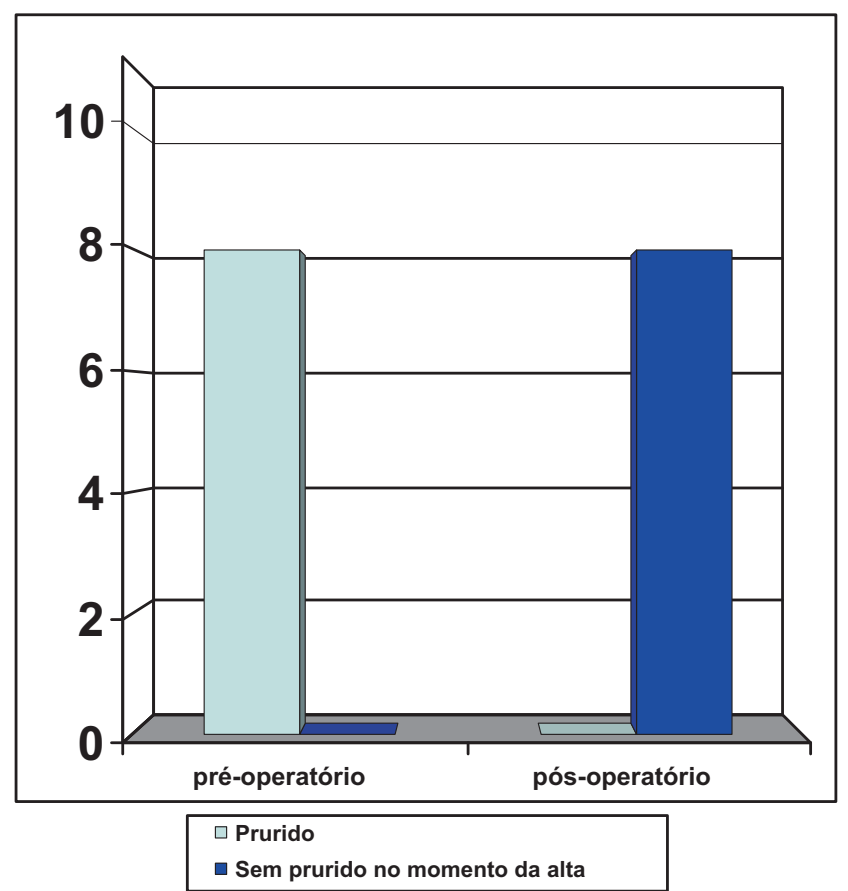

Gráfico 2 - Prurido pré e pós-operatório.

é realizada a drenagem bilateral (exceto com dois cateteres ou dois Stents) expondo o paciente ao risco de colangites de repetição. As complicações e mortalidade das abordagens nãocirúrgicas são notadamente menores.

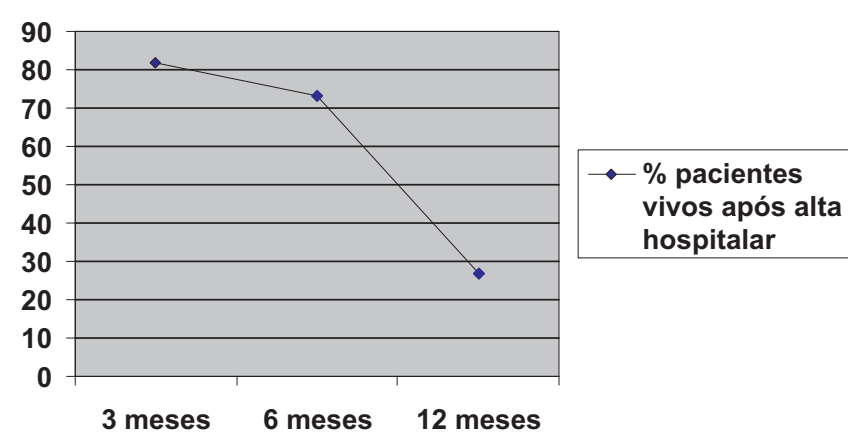

Gráfico 3 - Sobrevida após o procedimento.

A operação é indicada em pacientes que necessitam resolução no trans-operatório ${ }^{3}$. A morbidade e mortalidade estão de acordo com a literatura, variando de 6 a 30\%. Nenhuma complicação foi diretamente relacionada ao procedimen$\mathrm{to}^{2,3}$.

A hepatojejunostomia periférica não possibilita retorno completo das bilirrubinas aos níveis sangüíneos normais. Quando em único lobo, há o risco de colangite na árvore biliar do lobo contra-lateral ${ }^{3}$. A drenagem bilateral não apresenta maiores taxas de complicações ou colangite.

A hepatojejunostomia periférica demonstrou, pela sua eficácia, segurança, sangramento mínimo, rápida execução e mortalidade aceitável,ser uma boa opção como tratamento paliativo. Não ocorreram complicações diretamente relacionadas ao procedimento e foi possível uma melhora significativa da icterícia, alívio do prurido e sobrevida satisfatória.

\begin{abstract}
Background: This study shows the experience of our service with peripheral hepatojejunostomy. Methods: Between 2000 and 2005, eleven patients were submitted to peripheral hepatojejunostomy. Laparotomy in all patients had been performed as an attempt for curative resection. We analyzed age, gender, morbimortality, pre and post-operatory dosage of bilirubin, pre and post-operatory pruritus and survival. Results: The mean age of the patients was 67 years, six were female and five were male. Jaundice was present in $100 \%$ of cases, and pruritus in $80 \%$. Gallbladder neoplasm was diagnosed in six patients and cholangiocarcinoma in five. There were three intrahospital deaths. In preoperatory, mean values of total bilirubin was $19.33 \mathrm{mg} / \mathrm{dl}$ and of direct bilirubin was $16.81 \mathrm{mg} / \mathrm{dl}$, and in the post-operatory, $4.88 \mathrm{mg} / \mathrm{dl}$ and $3.64 \mathrm{mg} / \mathrm{dl}$, respectively. The eight discharged patients did not referred pruritus anymore, and their mean survival was eight months. All patients evoluted without jaundice and pruritus. Conclusions: Peripheral Hepatojejunostomy can be considered as capable to improve quality of life of the icteric patients. Surgery was demonstrated to be safe, with minimal blooding, rapid execution, and acceptable mortality rates. It was possible to improve significatively jaundice, pruritus, and to give a satisfactory survival.
\end{abstract}

Key words: Liver/surgery; Liver neoplasms; Palliative care.

\title{
REFERÊNCIAS
}

1. Pichlmayr R, Weimann A, Klempnauer J, Oldhafer KJ, Maschek $\mathrm{H}$, Tusch G, Ringe B. Surgical treatment in proximal bile duct cancer. A single-center experience. Ann Surg. 1996; 224(5):62838.

2. Su CH, Tsay SH, Wu CC, Shyr YM, King KL, Lee CH, Lui WY, Liu TJ, P'eng FK. Factors influencing postoperative morbidity, mortality, and survival after resection for hilar cholangiocarcinoma. Ann Surg. 1996; 223(4):384-94.

3. Schlitt HJ, Weimann A, Klempnauer J, Oldhafer KJ, Nashan B, Raab R, Pichlmayr R. Peripheral hepatojejunostomy as palliative treatment for irresectable malignant tumors of the liver hilum. Ann Surg. 1999; 229(2):181-6. 
4. Parc Y, Frileux P, Balladur P, Delva E, Hannoun L, Parc R. Surgical strategy for the management of hilar bile duct cancer. Br J Surg. 1997; 84(12):1675-9.

5. Longmire WP, Sandford MC. Intrahepatic cholangiojejunostomy with partial hepatectomy for biliary obstruction. Surgery. 1948; 24;264-76.

\section{Como citar este artigo:}

Coral RP, Rinaldi N, Bassols GF, Santos PR, Neves KR. Hepatojejunostomia periférica: uma alternativa técnica no tratamento paliativo das neoplasias malignas do hilo hepático. Rev Col Bras Cir. [periódico na Internet] 2008; 35(3). Disponível em URL: http:/ /www.scielo.br/rcbc

Endereço para correspondência:

Roberto Pellegrini Coral

Av. Pereira Passos, 562

Vila Assunção

91900-240 - Porto Alegre - RS

E-mail: coralrp@terra.com.br 